\title{
Alteration of Adaptive Immunity in a Colorectal Peritoneal Carcinomatosis Model
}

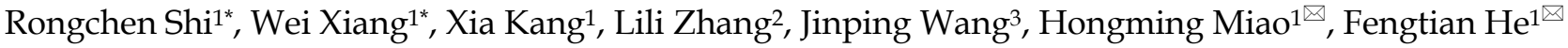 \\ 1. Department of Biochemistry and Molecular Biology, Third Military Medical University (Army Medical University), Chongqing 400038, China \\ 2. Department of Military Psychology, School of Psychology, Third Military Medical University (Army Medical University), Chongqing 400038, China \\ 3. Department of Neurology, Chongqing Emergency Medical Center, Chongqing 400014, China \\ ${ }^{*}$ Co-first authors \\ $\bowtie$ Corresponding authors: hmmiao@tmmu.edu.cn (H.M.M.), hefengtian06@aliyun.com (F.T.H.) \\ ( $)$ Ivyspring International Publisher. This is an open access article distributed under the terms of the Creative Commons Attribution (CC BY-NC) license \\ (https://creativecommons.org/licenses/by-nc/4.0/). See http://ivyspring.com/terms for full terms and conditions.
}

Received: 2018.06.18; Accepted: 2018.10.03; Published: 2019.01.01

\begin{abstract}
Colorectal cancer (CRC) usually gives rise to transcoelomic spread and ultimately causes peritoneal carcinomatosis (PC). However, mechanism studies, especially the immunological basis of colorectal PC, are rarely revealed due to lack of a suitable PC model. Here we selected a mouse colorectal cancer cell line MC-38 for intraperitoneal inoculation in the C57BL/6 mice to mimic the development of colorectal PC. We demonstrated that the injected CRC cells preferentially and rapidly migrated and colonized in the visceral fat tissues, but not in other visceral organs. With flow cytometric analysis, we found the proportions of spleen T cells and B cells were not affected by PC progression, while the ratios of blood CD4+ and CD8+ T cells were largely influenced. Especially, the quantity or activity of CD4+ and CD8+ T cells in visceral fats were intimately regulated by PC development. Taken together, we successfully constructed a colorectal PC model in immune-competent mice and revealed the alteration of adaptive immunity in PC development. Our study might potentiate the research and therapy strategies of colorectal PC.
\end{abstract}

Key words: peritoneal carcinomatosis, adaptive immunity, colorectal cancer

\section{Introduction}

Colorectal cancer (CRC) is the third most common malignancy, with a major cause of mortality by metastasis ${ }^{1-2}$. Besides the hematogenous and lymphatic routes, CRC usually gives rise to transcoelomic spread and ultimately causes peritoneal carcinomatosis (PC) $)^{3-5}$. PC is encountered in around $7 \%$ of patients at primary surgery, in $4 \%$ to $19 \%$ of patients during follow-up after curative surgery, in up to $40 \%$ to $80 \%$ of patients who succumb to CRC4.

In the past, the CRC patients with PC are considered as being in the terminal phase of their disease, and receive only supportive treatments, including the palliative surgery and systemic chemotherapy, achieving a survival rate not exceeding 7 months with 5-FU and Leucovorin ${ }^{6}$, reaching up to 23.4 month survival with Oxaliplatine and Irinotecan ${ }^{7}$. In the last decade, PC is considered as an initial loco-regional disease and renewed approaches for this disease are contributed by many research groups. The complete cytoreduction of macroscopic disease combined with hyperthermic intraperitoneal chemotherapy (HIPEC) has shown curative benefits on colorectal $\mathrm{PC}^{8-9}$, achieving overall survival rates up to 46 months and five-year survival rates up to $51 \% 7$.

The occurrence of colorectal PC relies on two important steps ${ }^{10}$. Firstly, the primary CRC cells need spread into peritoneal cavity. The primary invasive CRC could break through the bowel all and the cancer cells might fell off ${ }^{11}$. This case is encountered at primary surgery, with an incident rated around $7 \% 12$. The most frequent route for peritoneal dissemination comes from surgery-resulted rupture of bowel and vascular system ${ }^{4}$. The invading cancer cells in the lymphatic and blood vessels disseminate into the 
peritoneal cavity and targets a more adaptable microenvironment for malignant development. It's reported that this kind of PC cases account for up to $19 \%$ of postoperative patients ${ }^{4}$. Secondly, the disseminated cancer cells need target and colonize in a peritoneal tissue or organ, which could provide a pro-tumor microenvironment.

It's reported that the PC of gastrointestinal cancers and ovarian cancers prefers to grow in omentum majus. Some groups demonstrate, in a ovarian PC model, that fat tissues support tumor growth by providing the cancer cells with fatty acids ${ }^{13-14}$. However, few studies mentioned the immune status in PC progression, perhaps due to the lack of in vivo PC model with competent immunity. Subsequently, the exploration of immunotherapy for PC was restrained, although the $\mathrm{T}$ cell-based immunotherapy had obtained a great achievement in many terminal cancers ${ }^{15}$. In the present study, we set up a colorectal PC model in immune-competent mice and revealed the alteration of adaptive immunity in PC development. Our study might potentiate the research and therapy strategies of colorectal PC.

\section{Materials and Methods}

\section{Cell lines}

Mouse colorectal cancer cell lines MC-38 cells were provided by JENNIO Biological Technology (Guangzhou, China) and MC-38G (GFP-tagged MC-38 cells) were maintained in our research group. Those cells were maintained in Dulbecco's modified Eagle's medium (DMEM) high glucose, supplemented with $10 \%$ fetal bovine serum (FBS), penicillin $(100 \mathrm{U} / \mathrm{ml})$ and streptomycin $(100 \mathrm{ug} / \mathrm{ml})$, in a $5 \%$ $\mathrm{CO}_{2}$ atmosphere at $37^{\circ} \mathrm{C}$.

\section{Animal model}

All the animal experiments were approved by the Institutional Animal Care and Use Committee at Third Military Medical University (TMMU), and all the experiments were performed in accordance with the "Guide for the care and use of laboratory animals" published by the US NationalInstitutes of Health (publication no. 85-23, revised 1996). All the mice were housed in a pathogen-free facility with a 12-h light, 12-h dark cycle in TMMU. All the mice were provided with food and purified water ad libitum. Each cage contained no more than 5 mice. MC-38 cells and MC-38G cells $\left(1 \times 10^{6}\right.$ cells in $0.1 \mathrm{ml}$ PBS) were intraperitoneally injected into abdominal cavity of 6 week-old male C57BL/ 6 mice. Then the tumorinoculated mice were observed dynamically. The pathological observation of PC development including tumor infiltration and systemic parameters were performed on day $0,5,10,15$ and 20. The alteration of T cells and B cells was evaluated on day $0,3,5,7$ and 9. The production of IFN- $\gamma$ in T cells was measured on day $0,5,10$ and 15 .

\section{HE staining}

Organs and tissue were harvested, formalinfixed, and processed into paraffin blocks, and then sections were cut and stained with hematoxylin and eosin (C0105, Beyotime, China).

\section{Immunofluorescence staining}

All tissue samples were fixed and processed into paraffin blocks. After dewaxing, antigen repair (10 mmol/L sodium citrate for $5 \mathrm{~min}$ in a pressure cooker) and removing endogenous enzymes in Hydrogen Peroxide Block, the sections were incubated with the primary antibodies anti-GFP (ab13970, Abcam, UK, the dilution ratio was 1:500) and anti-Perilipin (ab3526, Abcam, UK, the dilution ratio was 1:200) at $4^{\circ} \mathrm{C}$ overnight. Then the samples were incubated in secondary antibody (703-545-155, Jackson ImmunoResearch, USA; ab150074, Abcam, UK, the dilution ratio was 1:500) for $1-2 \mathrm{~h}$ at room temperature in the dark. After rinsing three times in 1X PBS for $5 \mathrm{~min}$ each, the slides were counterstained with Hoechst (C1026, Beyotime, China, the dilution ratio was 1:500) to reveal cell nuclei. Finally, fluorescence microscopy was used to evaluate specimen.

\section{Immunostaining and fluorescence-activated cell sorting (FACS)}

Mice were sacrificed by $\mathrm{CO}_{2}$. The spleen was dissected and gently rubbed between the two rough sides of frosted slides (\#FSL006, Beyotime, Shanghai, China). Splenic samples were isolated by gently lapping spleens over a 75- $\mu$ m filter (F513442, Sangon Biotech, China), and then washing in FACS buffer (PBS with 0.5\% endotoxin-free FBS, $2 \mathrm{mM}$ EDTA, and $25 \mathrm{mM}$ HEPES). After spinning down for $5 \mathrm{~min}$ at $500 \mathrm{~g}$, erythrocytes were removed by ACK lysis buffer (R1010, Solarbio, China) for 5 minutes. Then the reaction was stopped by adding equivoluminal PBS buffer and splenocytes were collected by centrifugation. Finally, cell pellet were resuspended with FACS buffer.

Circulating blood samples were collected using blood collection tubes (171001, KANG JIAN, China) to prevent clotting. After using mouse peripheral blood lymphatic separation kit (\#P8620, Solarbio, China), cells were collected and lysed in ACK lysis buffer as described above. In the same way, blood samples were resuspended in FACS buffer.

Adipose tissue were resected from mice and mechanically dissociated with ophthalmic forceps into about one cubic millimeter and then digested with $25 \mathrm{ml}$ Roswell Park Memorial Institute (RPMI) 
1640 medium, supplemented with 3\% FBS and $1 \mathrm{mg} / \mathrm{ml}$ collagenase IV ( A004186, Sangon Biotech, China) for $45 \mathrm{~min}$ at $37^{\circ} \mathrm{C}$. Meanwhile the solution was vibrated by magnetic stirrer. After dissociation, adipose tissue suspensions were filtered through a $75-\mu \mathrm{m}$ filter, and were spun down for $10 \mathrm{~min}$ at $500 \mathrm{~g}$. Then adipose tissue samples were lysed with ACK buffer as above, and then resuspended in FACS buffer.

At last, after staining with antibodies at $4{ }^{\circ} \mathrm{C}$ for 45 min followed by 2 washes in FACS buffer, the cells were resuspended in FACS buffer for FACS analysis (FACSVerse C6, BD Biosciences). The antibodies included APC anti-mouse CD45 antibody (\#103112, Biolegend), PE anti-mouse CD90.2 antibody (\#105308, Biolegend), PerCP/Cy5.5 anti-mouse CD8a antibody (\#100734, Biolegend), and APC/Cy7 anti-mouse CD4 (\#105308, Biolegend).

\section{Treatment of antibodies in vivo}

The antibody treatment was performed as described previously ${ }^{16}$. Briefly, MC-38 cells $\left(1 \times 10^{6}\right.$ cells in $0.1 \mathrm{ml}$ PBS) were intraperitoneally injected into abdominal cavity of 6 week-old male C57BL/ 6 mice. Then, the MC-38 tumor-bearing mice were treated with monoclonal antibodies anti-CD8 (MAB116, Novus Biologicals) or IgG (MAB002, Novus Biologicals) as control. Tumor cells were inoculated on day 1. Each mouse was treated 3 times (days 4, 7 and 10) via the tail vein at a dose of $100 \mathrm{ug} /$ day. The tumor nodes in visceral fats were checked on day 15.

\section{Statistical analysis}

All analyses were performed using GraphPad Prism 5. All data were expressed as the mean \pm s.e.m., and were analyzed using two-tailed unpaired T-test. For all statistical analyses, * indicates $P<0.05$, ** indicates $P<0.01$, and *** indicates $P<0.005$.

\section{Results}

\section{CRC cells mainly target the visceral fat tissues}

To mimic the disseminated CRC cells in peritoneal cavity, a mouse colorectal cancer cell line MC-38 was employed for intraperitoneal inoculation in C57BL/ 6 mice. The PC progression was observed dynamically. We demonstrated that the PC nodes were visible 15 days post inoculation in our experimental system (Figure 1A-E). Interestingly, the PC nodes mainly located in visceral fats, such as omentum majus (Figure 1B), mesenterium (Figure 1C) and epididymal fats (Figure 1E). After 20 days of inoculation, the PC nodes could be observed in almost all the visceral fat tissues, even in perirenal fats (Figure 1D). Meanwhile, the body weight of tumor bearing mice was significantly lost in comparison with the tumor-free mice (Table 1). However, the weight of organs like spleen, kidney, lung and liver didn't differ notably between the PC and control group (Table 1). It should be pointed out that the tumor-bearing mice usually died around 25 days post tumor injection (data not shown).

Table 1. The weight of visceral organs in the PC model

\begin{tabular}{lll}
\hline Parameters & PBS & MC-38 \\
\hline BW $(\mathrm{g})$ & $20.66 \pm 0.34$ & $17.20 \pm 0.75^{* *}$ \\
Spleen weight $(\mathrm{g})$ & $0.13 \pm 0.0260$ & $0.13 \pm 0.0118$ \\
Spleen to BW ratio (\%) & $0.61 \pm 0.0013$ & $0.74 \pm 0.0007$ \\
Kidney weight (g) & $0.30 \pm 0.0215$ & $0.33 \pm 0.0196$ \\
Kidney to BW ratio (\%) & $1.45 \pm 0.0010$ & $1.89 \pm 0.0011^{* *}$ \\
Lung weight (g) & $0.14 \pm 0.0090$ & $0.15 \pm 0.0046$ \\
Lung to BW ratio (\%) & $0.69 \pm 0.0004$ & $0.86 \pm 0.0003^{* *}$ \\
Liver weight (g) & $1.26 \pm 0.1081$ & $1.45 \pm 0.0828$ \\
Liver to BW ratio (\%) & $6.10 \pm 0.0052$ & $8.42 \pm 0.0048^{* *}$ \\
\hline
\end{tabular}

Six-week-old mice were intraperitoneally inoculated with MC- 38 cells $\left(1 \times 10^{6}\right.$ cells in $100 \mathrm{ul} \mathrm{PBS}$ for each mouse) or PBS as control. 20 days later, the visceral organs were dissected and weighed. Data are mean \pm s.e.m. $\left(n=5, * * P<0.01,{ }^{* * *} \mathrm{P}<0.005\right.$, student's t-test)

To further confirm the target of CRC cells in PC development, we dissected the visceral organs, such as guts, livers, lungs, kidneys and spleens. No tumor nodes were observed in gross anatomy of those organs 20 days post of tumor inoculation, except the mesenterium attached to guts (Figure 2A). We also didn't find any tumor lesions in spleens, kidneys, lungs or livers from tumor bearing mice with pathological observation (Figure 2B).

\section{CRC cells quickly invaded into the visceral fats}

In the present PC model, CRC cells mainly migrated to the visceral fat tissues. As shown in Figure 3A, the epididymal fats were isolated and easy to investigate. Thus we choose this fat tissue to dynamically assess the progression of colorectal PC. In consistence, the PC nodes in epididymal fats were visible 15 days post tumor injection (Figure 3A). With HE staining, we could find CRC cell infiltration on day 10 after setup of the present PC model (Figure 3B). To further characterize the detailed metastasis in epididymal fat, the GFP-tagged MC-38 cells were used to set up the PC model. We demonstrated, on day 5 post PC model setup, that the MC-38 tumor cells were infiltrated in the epididymal fat (Figure 3C). This result indicated that the inoculated CRC cells could rapidly migrate into the visceral fat tissues.

\section{The status of systemic adaptive immunity in the colorectal PC model}

Adaptive immunity plays a crucial role in the development of tumors, and T cell-based immunotherapy is increasingly being approved for clinical use $^{15,17}$. The $\mathrm{CD}^{+} \mathrm{T}$ cells, also known as cytotoxic $\mathrm{T}$ lymphocyte, can directly attack foreigners such as 
bacteria, viruses, and cancer cells once they become activated $^{18}$. By contrast, CD4 ${ }^{+} \mathrm{T}$ cells (mature T-helper cells) mainly organize an immune response through indirectly recruiting other immune cells ${ }^{19}$. Therefore, we further measured the adaptive immune alteration in spleen and blood with immunostaining and FACS. We found the ratios of $T$ cells and $B$ cells in spleens were not altered significantly in response to tumor injection (Figure 4A-B). Further, the spleen CD4 and CD8 subsets of T cells were also assed and no obvious differences were observed after PC setup (Figure 4C).

Noteworthily, numbers of blood $\mathrm{T}$ cells underwent a quick decrease with PC development, while blood B cells didn't alter notably (Figure 5A-B). In details, the ratios of blood CD4+ T cells decreased slightly over time after tumor inoculation, whereas the ratios of $\mathrm{CD} 8+\mathrm{T}$ cells increased significantly (Figure 5C). In comparison with CD8, the amount of blood CD4+ T cells continuously decreased with PC progression (Figure 5B-C).
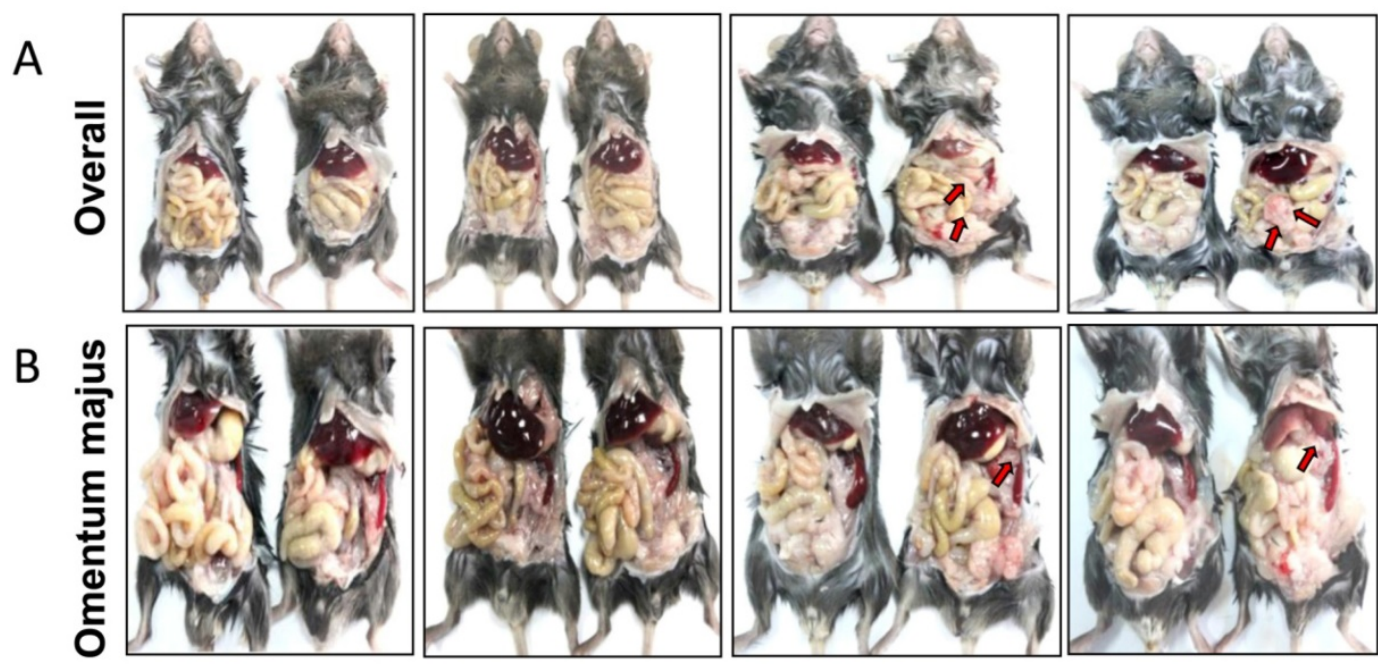

C
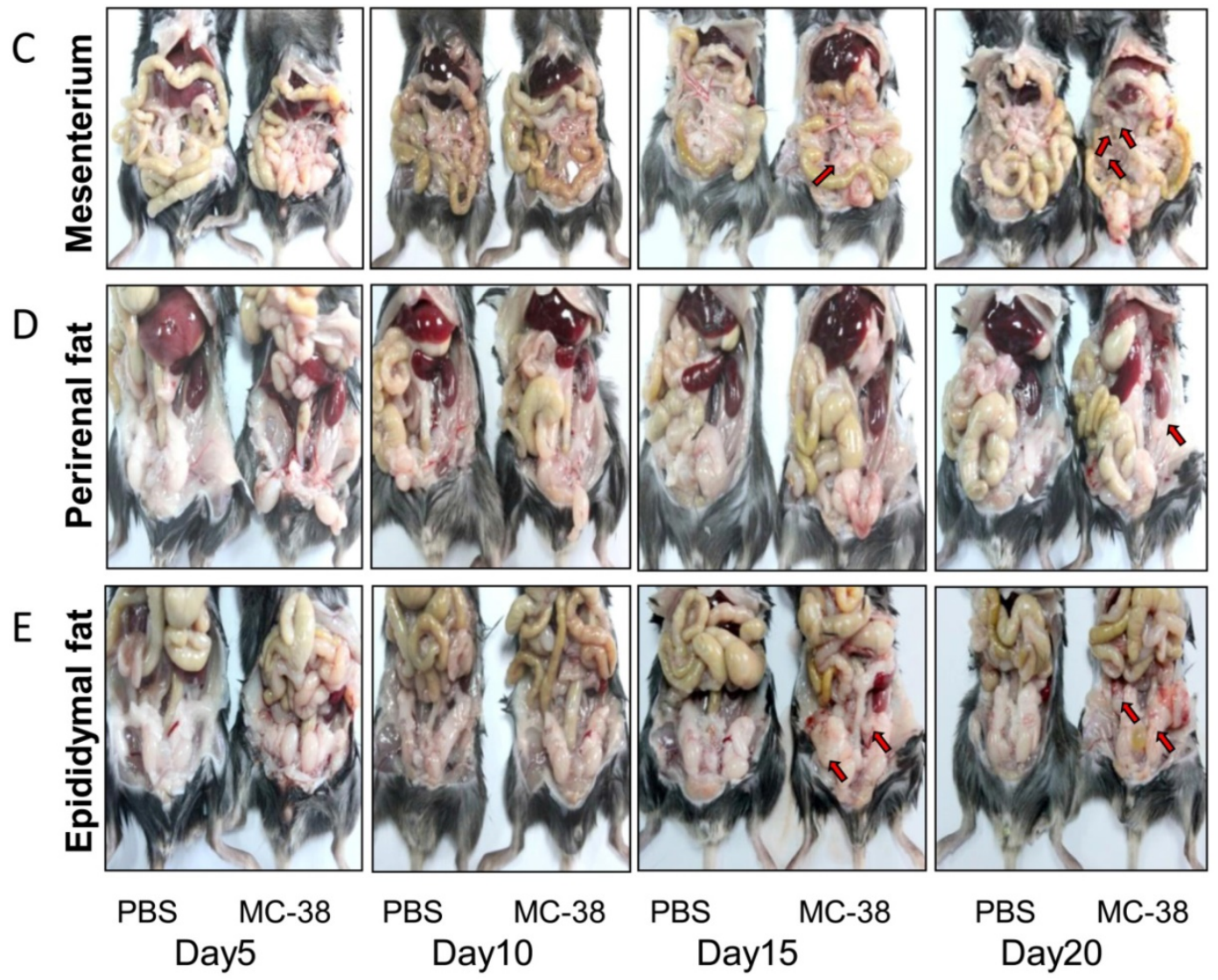

Figure 1. Peritoneal CRC cells mainly target the visceral fat tissues. (A) The overall status of colorectal PC development in mice. Six-week-old mice were intraperitoneally inoculated with MC-38 cells $\left(1 \times 10^{6}\right.$ cells in 100 ul PBS for each mouse) or PBS as control, and PC progression was observed dynamically. The red arrows indicated tumor nodes. Representative images were displayed. $(n=5)(B-E)$ PC progression in the omentum majus $(B)$, mesenterium $(C)$, perirenal fat $(D)$ and epididymal fat $(E)$ of the mice described in (A). The red arrows indicated tumor nodes. Representative images were displayed. $(n=5)$ 
A

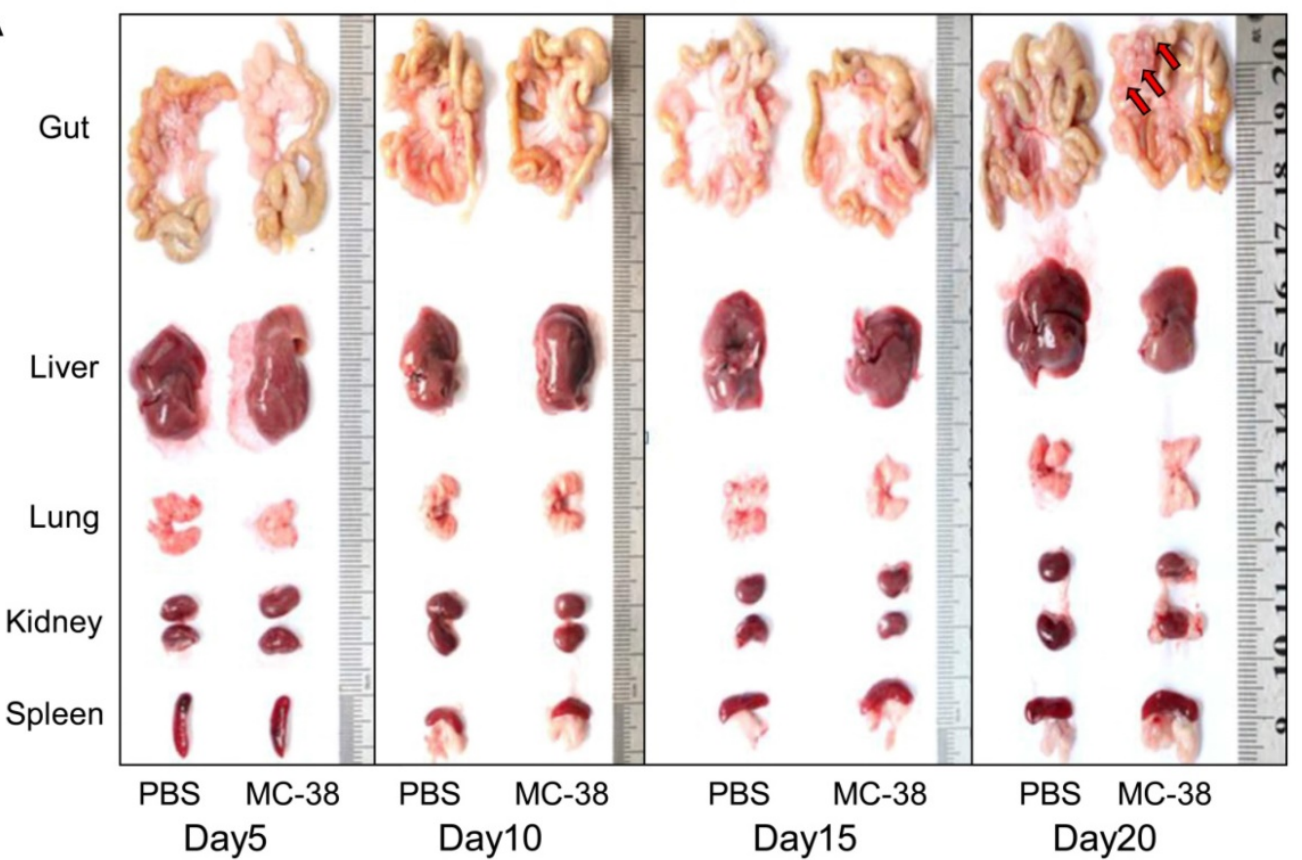

B
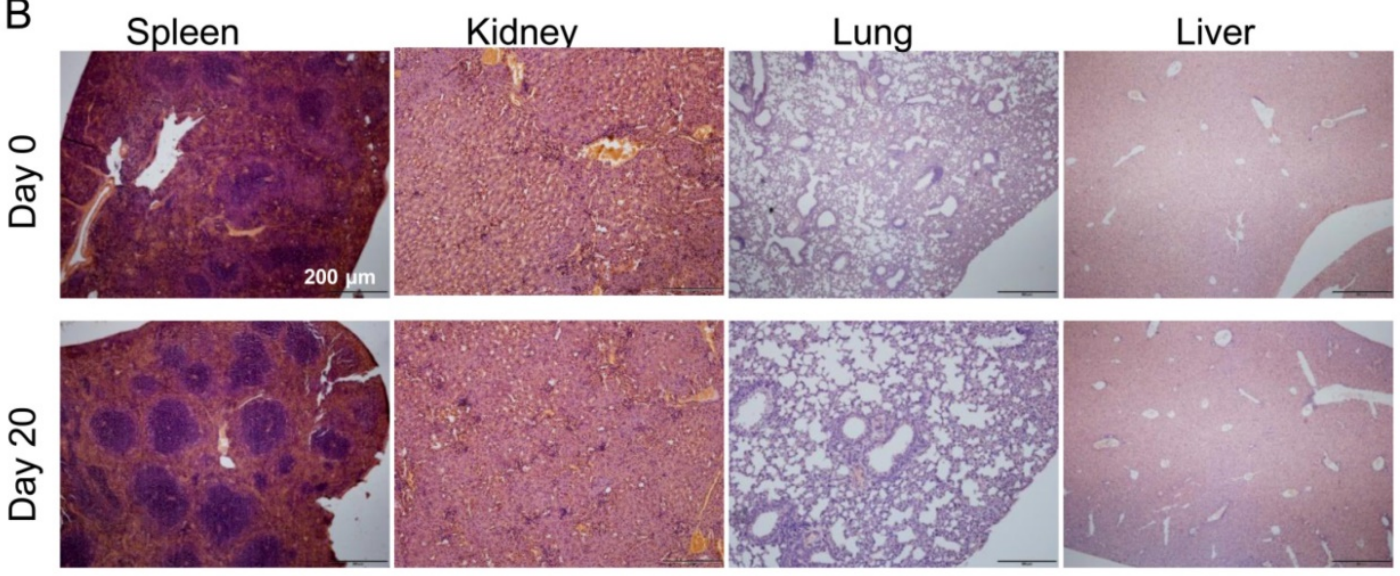

Figure 2. Peritoneal CRC cells doesn't target visceral organs. (A) The gross anatomy of visceral organs from MC-38 PC models as indicated above in (Figure $1 \mathrm{~A}$ ). Representative images of the fresh organs were displayed. $(n=5)(B)$ H\&E staining of the visceral organs from PC models described above in (A). Representative images were displayed. Scale bars, $200 \mu \mathrm{m}$. $(n=5)$

\section{The status of local adaptive immunity in the colorectal PC model}

Comparing with the systemic immunity, the local immunity in tumor microenvironment appears more effective. Therefore, we further investigated the adaptive immunity alteration in visceral fats and the epididymal fat was selected as a representative. With FACS analysis, we demonstrated that the amount of $T$ cells was continuously increased over time after tumor inoculation, while the B cells altered modestly (Figure 6A-B). Further, we found the ratio of CD4+ T cells increased on day 5 after tumor injection, while declined to basal levels quickly (Figure 6C). More interestingly, the ratio of fat $\mathrm{CD} 8+\mathrm{T}$ cells was decreased on day 5 after tumor inoculation and quickly restored and increased over time (Figure 6C). Combining with the alteration of total $\mathrm{T}$ cells in fats, we concluded that the amount of fat CD8+ T cells was continuously increased in the process of PC development.

It's reported that the tumoricidal $\mathrm{T}$ cells would be exhausted with cancer progression. The exhausted $\mathrm{T}$ cells expressed less IFN- $\gamma$ than the functional $\mathrm{T}$ cells ${ }^{20}$. Thus we further detected the IFN- $\gamma$ levels in fat $\mathrm{T}$ cells dynamically in the PC model. We found that the levels of IFN- $\gamma$ in CD4+ $\mathrm{T}$ cells increased by around 2 fold on day 10 after tumor injection and then began to drop, as compared to tumor-free mice (Figure 7A-B). However, the IFN- $\gamma$ levels in fat CD8+ $\mathrm{T}$ cells fluctuated modestly over time (Figure 7C). It's interesting that the levels of IFN-Y in CD8+ T cells were much higher than that in CD4+ T cells (Figure 7B-C). 
According to the alteration of $\mathrm{T}$ cell quantity and quality in the fat tissues, we presumed that the T cells were not exhausted at least before day 10 and that $\mathrm{CD} 8+\mathrm{T}$ cell might be a major regulator of tumor immunity in our colorectal PC model. Next, we used CD8 antibodies to eliminate CD8+ $\mathrm{T}$ cells to investigate the role of CD8+ T cells in colorectal PC progression. We demonstrated that treatment of CD8 antibodies largely potentiated the development of MC-38 cell-based PC (Figure 8A-B). Those results indicated that PC development could be modulated by immunotherapy.

\section{A}
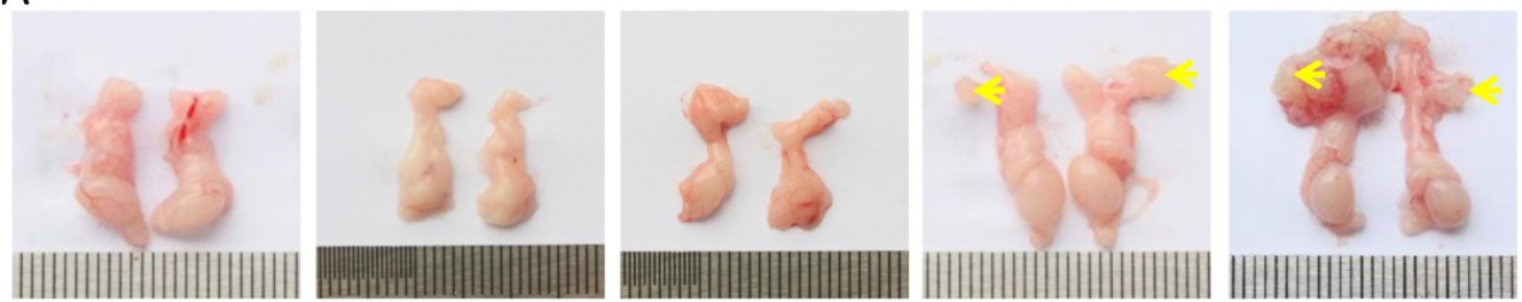

Day0

Day5

Day10

Day15

Day20

B

Day0

Day5

Day10

Day15

Day20
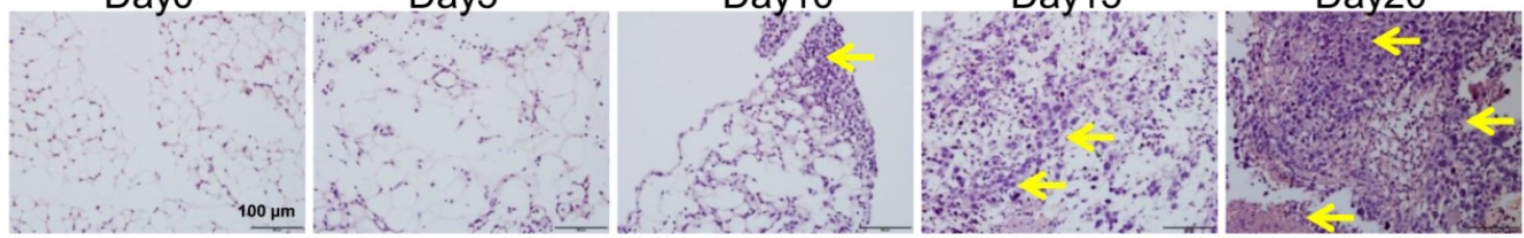

C

\section{Day5}

Day10
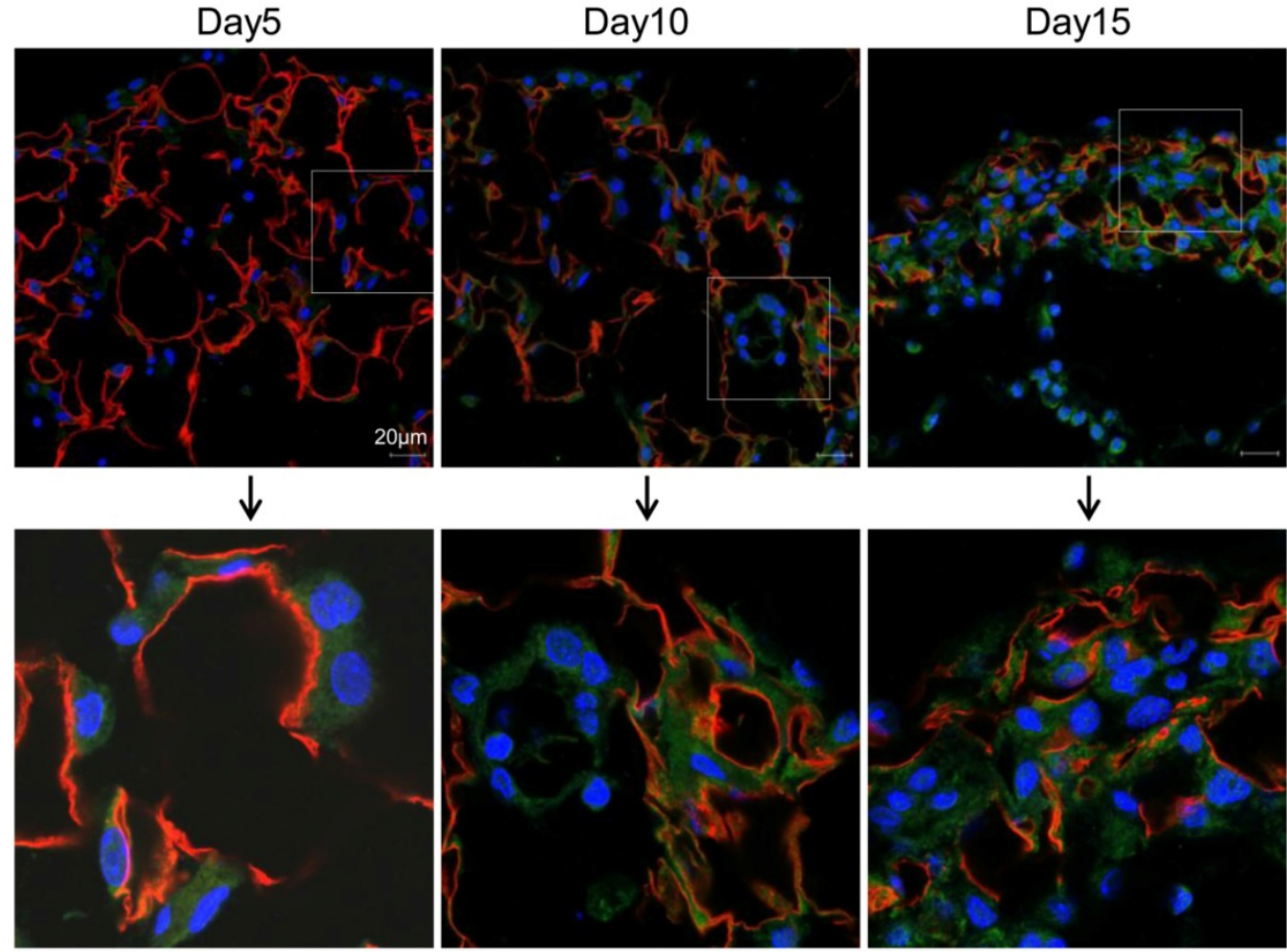

Perilipin/GFP/Hoechst

Figure 3. CRC cells quickly invade into the visceral fats. (A) The gross anatomy of the epididymal fats in $M C-38$ PC models as described above in (Figure $1 \mathrm{~A}$ ). Representative images were displayed. The arrows indicated the tumor nodes. $(n=5)(B)$ H\&E staining of the epididymal fat tissues described in $(A)$. The arrows indicated tumor lesions. Shown were representative images. Scale bars, $100 \mu \mathrm{m} .(n=5)(C)$ The rapid infiltration of CRC cells in the epididymal fat. Six-week-old mice were intraperitoneally injected with GFP-tagged MC-38 cells $\left(1 \times 10^{6}\right.$ cells in 100 ul PBS for each mouse) or PBS as control. The epididymal fat was collected dynamically and stained with GFP (Green) or perilipin (red) antibodies by immunofluorescence analysis. The nucleus was visualized by hoechst staining (Blue). Representative images were shown. Scale bars, $20 \mu \mathrm{m}$. ( $\mathrm{n}=5$ ) 

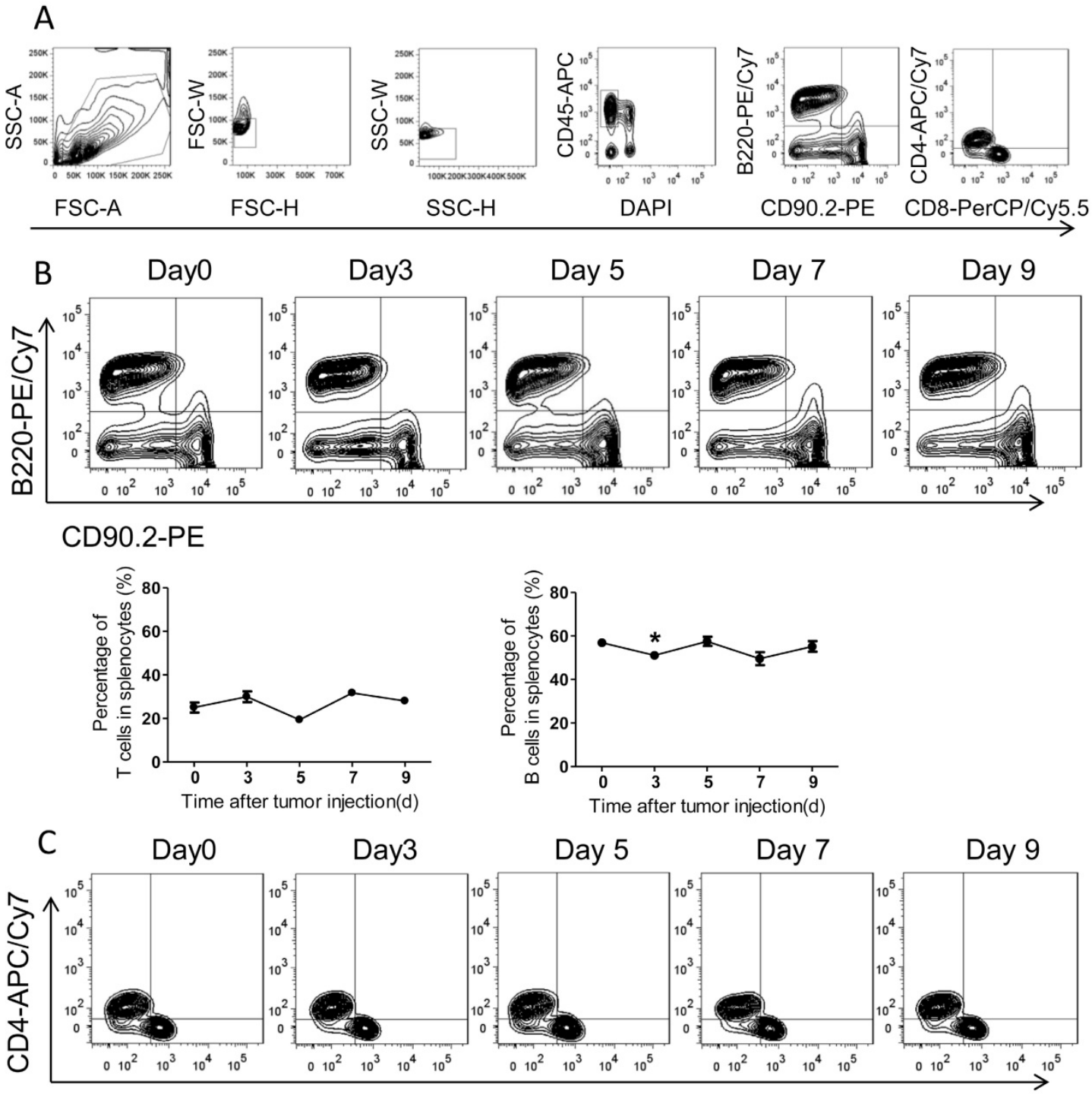

CD8-PerCP/Cy5.5
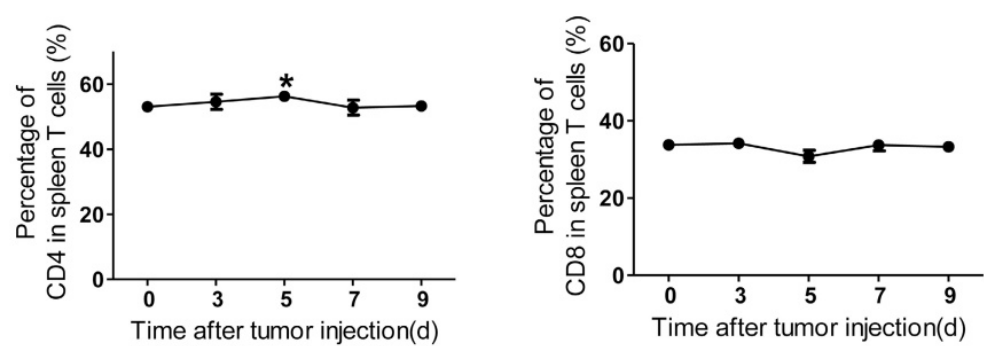

Figure 4. The adaptive immunity in spleens of the colorectal PC model. (A) FACS gating strategy for spleen T/B cells. Debris and doublets were removed, and then the spleen T/B cells were assessed as DAPI-CD45+CD90.2+/B220+. The levels of T/B cells and CD8+/CD4+ T cells were also measured. (B-C) The levels of spleen T/B cells at different time points. Six-week-old mice were intraperitoneally injected with MC-38 cells. Then the splenocytes were isolated for flow cytometric analysis of T/B cells (B) and CD8+/CD4+ T cells (C). Shown are representative images. Data are mean \pm s.e.m. $(n=3, * P<0.05$, student's t-test)

\section{Discussion}

By intraperitoneal inoculation of MC-38 cells, we set up a colorectal PC model in mice. We demonstrated that the CRC cells mainly migrated and colonized in the visceral fat tissues. With the analysis of adaptive immunity, we found that $\mathrm{T}$ cells were intimately altered with the development of PC. Thus we provided an ideal colorectal PC model for cancer and immunology research.

The present PC model can mimic the characteristics of colorectal PC in clinics. Besides, gastrointestinal and ovarian cancer PC could also be mimicked by intraperitoneal inoculation of corresponding cancer cells. It's reported that ovarian cancer cells were also predominantly colonized in visceral 
fats such as omentum majus, in mouse PC models ${ }^{13}$. It's really interesting that visceral fats are the major targets of PC in different types of cancers. The mechanisms for the attractive and supportive effects of fat tissues on peritoneal cancer cells need further to be explored. After that, potential targets for preventing colorectal PC might be revealed.

It should be pointed out that a number of PC models have been established by a number of previous and recent studies with different purposes. For general research on cancer cell biology, the PC models are mainly constructed in the nude mice by intraperitoneal inoculation of any types of cancer cells regardless of species ${ }^{21-22}$. Especially, some human colorectal cancer cell lines could be implanted in nude mice to test drug effects ${ }^{21-22}$. Besides, the murine MC-38 or CT-26 cell-based colorectal PC models were also established in the immune competent mice in the present or other studies ${ }^{23-25}$. More interestingly, the cancer PC models were also reported in rats $^{26}$ and rabbits ${ }^{27}$. However, few studies investigated the basic immunological changes with the PC development.

A

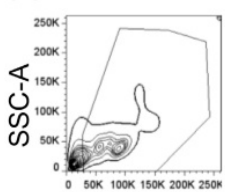

FSC-A

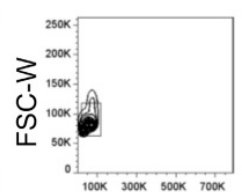

FSC-H

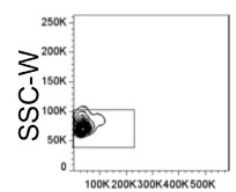

SSC-H
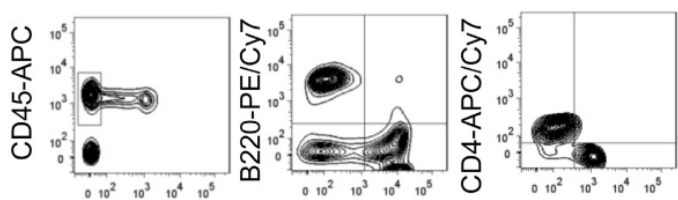

DAPI CD90.2-PE CD8-PerCP/Cy5.5

$\mathrm{B}$

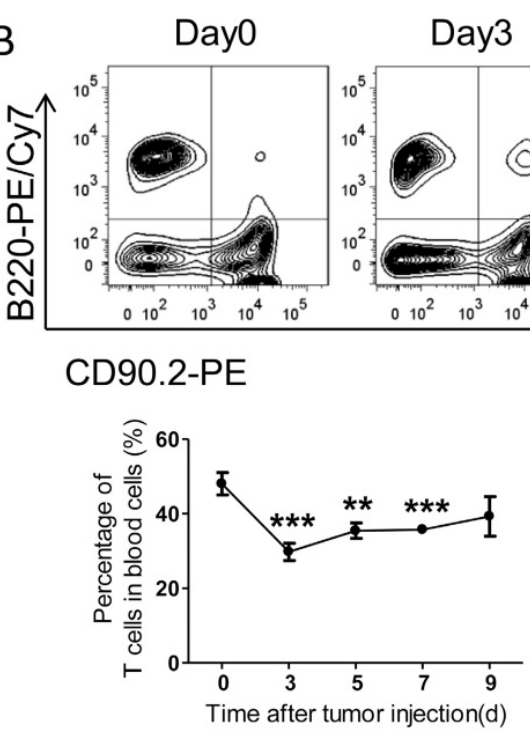

Day 5

Day 7

Day 9
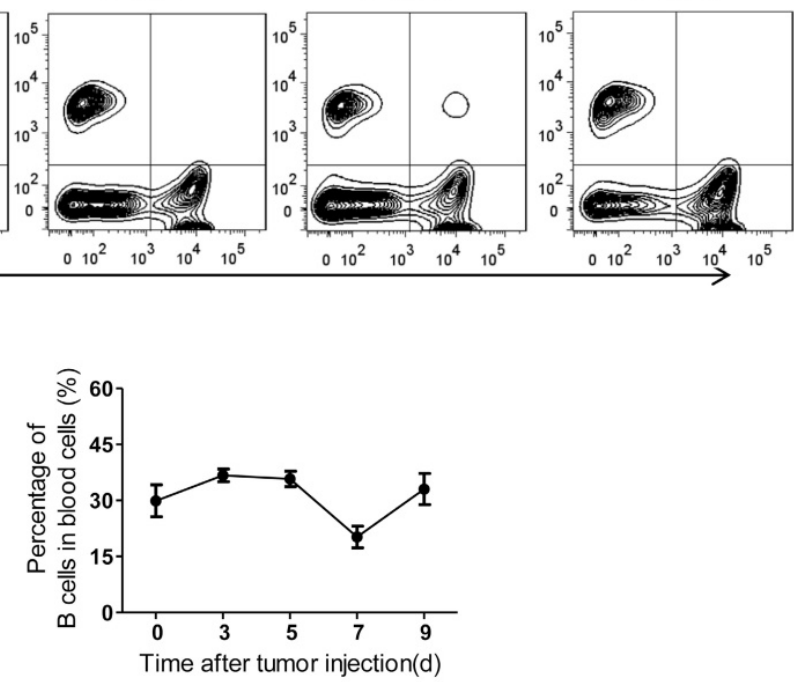

C

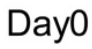

Day3

Day 5

Day 7
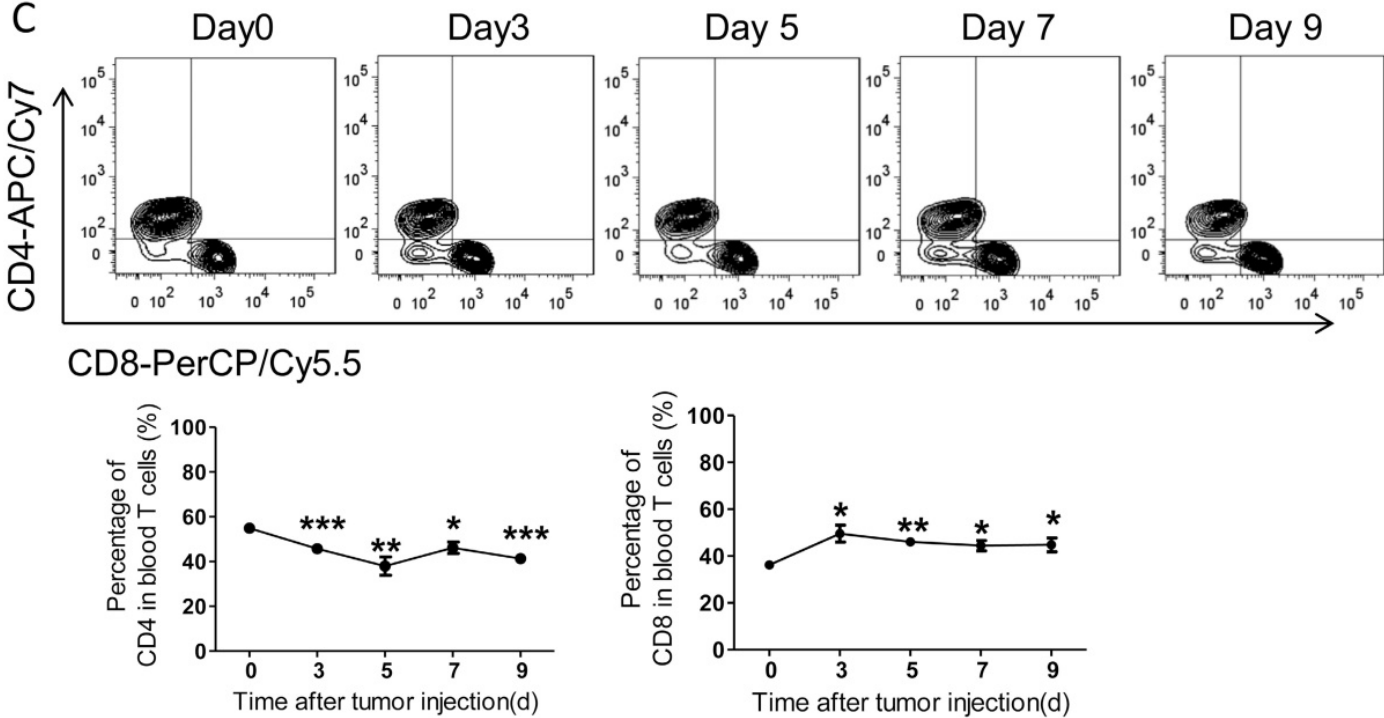

Figure 5. The adaptive immunity in blood of the colorectal PC model. (A) FACS gating strategy for blood T/B cells as described above in Figure 4A. (B-C) The levels of blood T/B cells at different time points. Blood of the mice described in Figure 4A was collected for flow cytometric analysis of T/B cells (B) and CD8+/CD4+ $T$ cells (C). Representative images were displayed. Data are mean \pm s.e.m. $\left(n=3, * P<0.05,{ }^{*} * P<0.01,{ }^{*} * * P<0.005\right.$, student's t-test) 


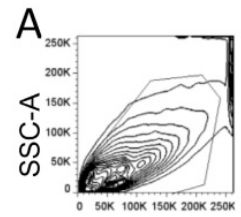

FSC-A

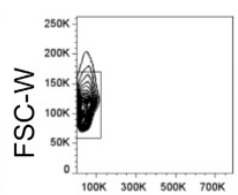

FSC-H

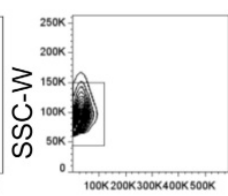

SSC-H

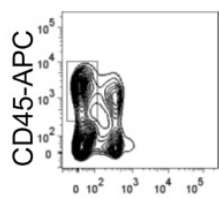

DAPI

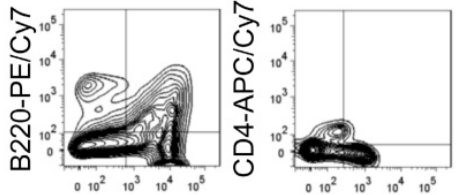

CD90.2-PE CD8-PerCP/Cy5.5

B

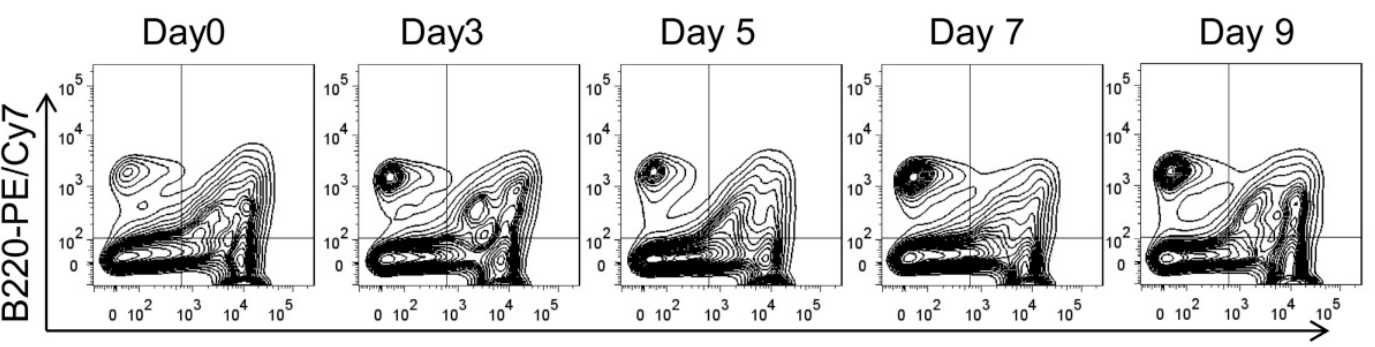

CD90.2-PE
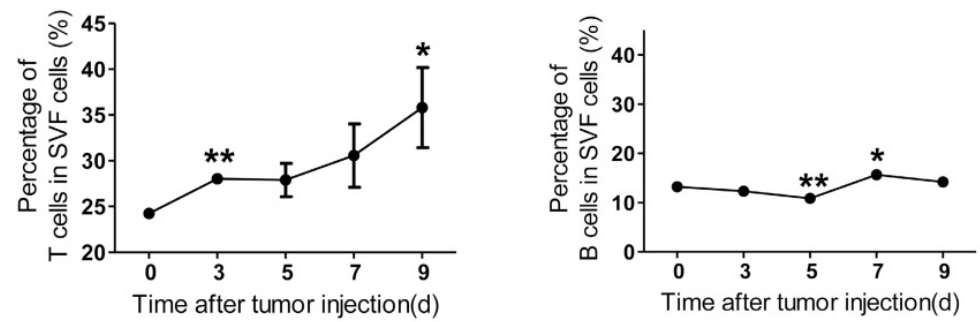

C Day0 Day3 Day 5 Day 7

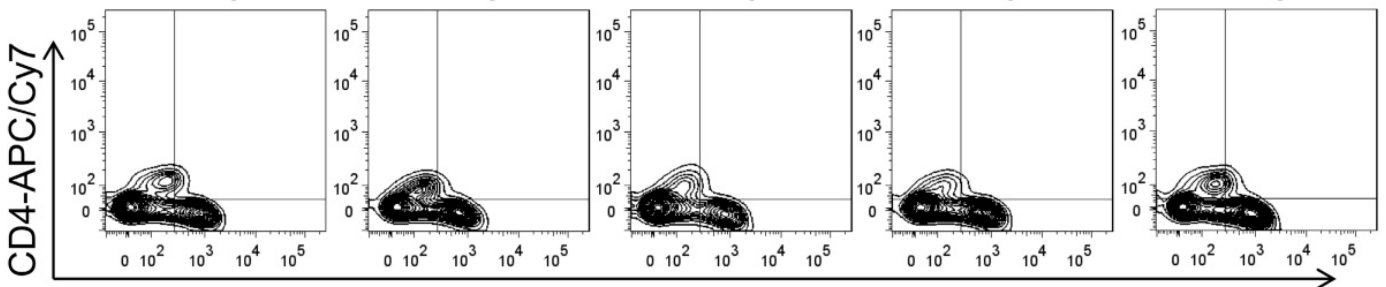

CD8-PerCP/Cy5.5
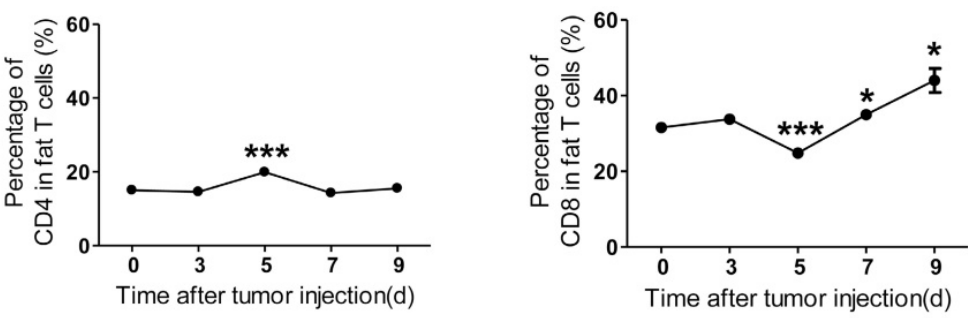

Figure 6. The adaptive immunity in epididymal fats of the colorectal PC model. (A) FACS gating strategy for epididymal fat T/B cells as described above in Figure $4 \mathrm{~A}$. (B-C) The levels of T/B cells in epididymal fats. The mice were treated as above, and then the adipose stromal cells were isolated for flow cytometric analysis of T/B cells (B) and CD8+/CD4+ T cells (C). Shown are representative images. Data are mean \pm s.e.m. $(n=3, * P<0.05, * * P<0.01$, ***P $<0.005$, student's t-test)

The previous studies suggested that adipocytes derived fatty acids might be the major resources of substances and energy for the development of cancer cells ${ }^{13}$, 28-29. However, those studies were mainly performed in PC models of immune-deficient mice. Actually, cancer progression is accompanied by immune alteration. Immunotherapy (such as PD1 and PDL-1 blockage ${ }^{30-31}$ ) has shown a great potential in cancer treatment. Our colorectal PC model was constructed in the immune complement mice, providing an ideal chance for immune studies. In the present study, we demonstrated that the quantity and quality of $\mathrm{T}$ cells in fat tissues altered dynamically with colorectal PC development. We speculated that modulation of $\mathrm{T}$ cell activity might be a good way to treat colorectal PC. The present model could be used for functional screening of immunotherapy-related drugs. The colorectal PC progressed quickly in our experimental model, making related studies rather convenient and reproducible. 

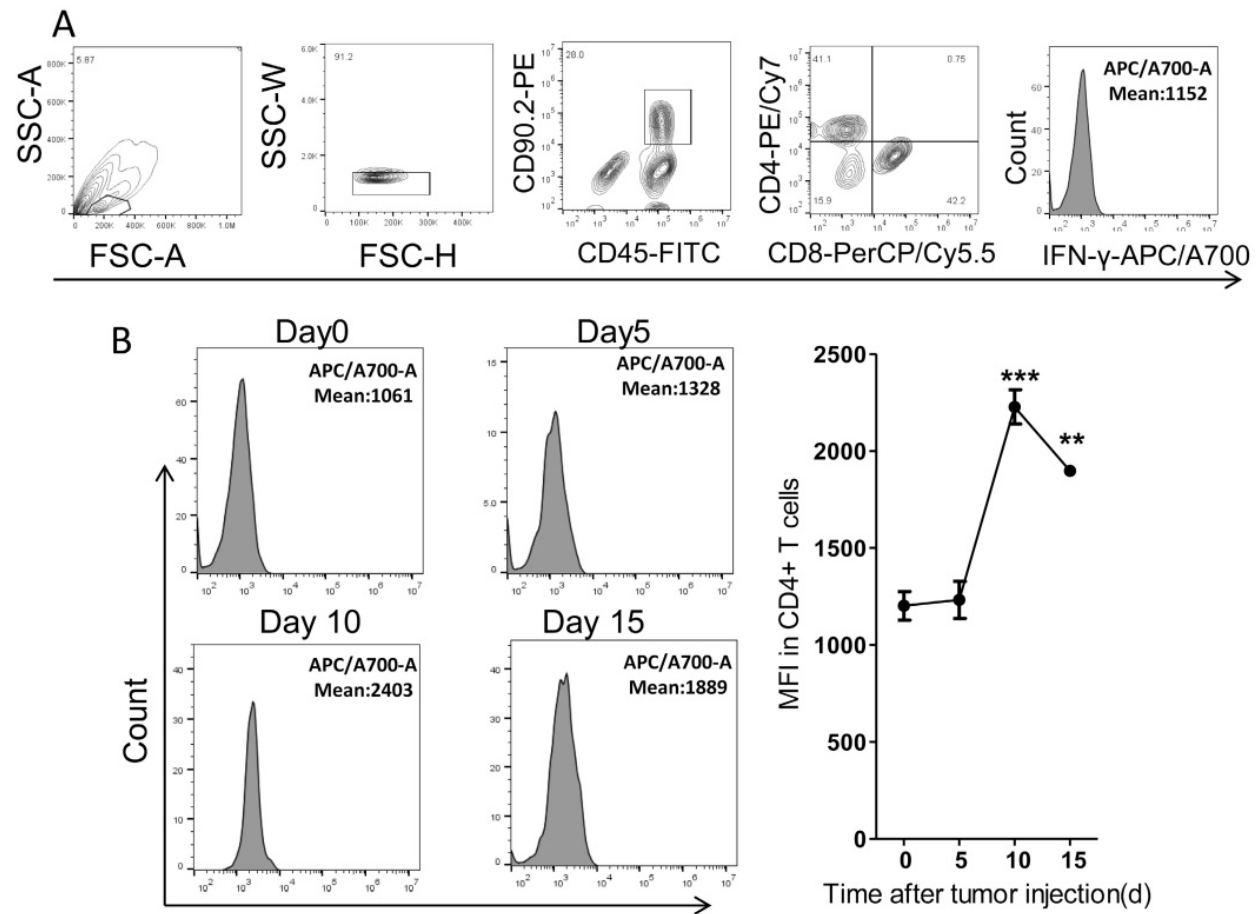

IFN- $\mathrm{Y}-\mathrm{APC} / \mathrm{AF} 700$

C

Day0

Day5

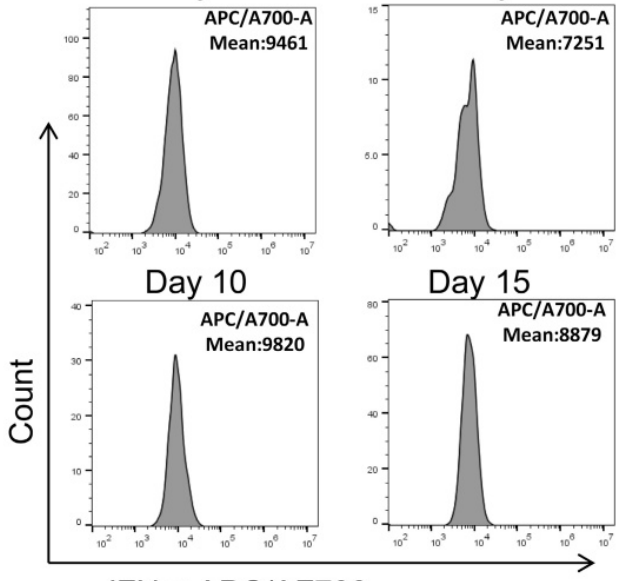

IFN-y-APC/AF700

Figure 7. The expression of IFN-Y in Fat T cells of the colorectal PC model. (A) FACS gating strategy for the measurement of IFN- $\gamma$. Debris and doublets were removed, and then the CD4+ T cells were assessed as DAPI-CD45+CD90.2+CD4+ and the CD8+ T cells were assessed as DAPI-CD45+CD90.2+CD8+. The average fluorescence degree indicating the level of IFN- $\gamma$ was measured. (B-C) The average fluorescence degree of IFN- $\gamma$ in $T$ cells. Six-week-old mice were intraperitoneally injected with MC-38 cells and then the adipose stromal cells were isolated for flow cytometric analysis of IFN-y levels in CD4+ T cells (B) or CD8+ T cells (C). Shown are representative images. Data are mean \pm s.e.m. $(n=3, * P<0.05, * * P<0.01$, $* * * P<0.005$, student's t-test)
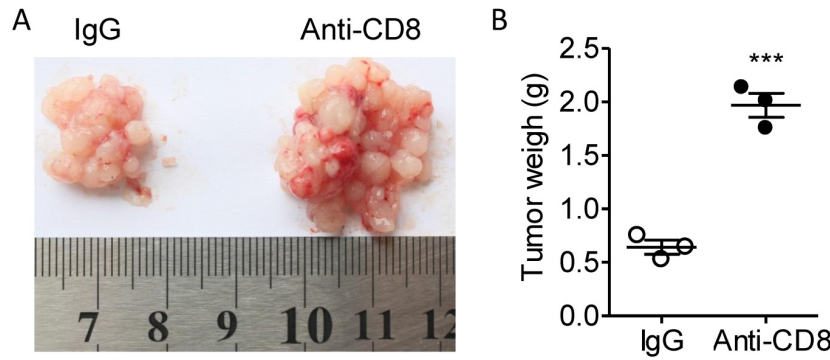

Figure 8. Anti-CD8 treatment potentiates the PC progression. (A) Six-week-old male C57BL/6 mice were intraperitoneally inoculated with MC-38 cells and then treated with anti-CD8 antibody or IgG as a control. The tumor nodes in visceral fats of each mouse were observed on day 15. Representative images were displayed. (B) The weight of tumor nodes in visceral fats as described above in (A). The data represent the means \pm s.e.ms. $(n=3, * * * P<0.005$, Student's t-test).
In all, here we provided a convenient and reliable colorectal PC model in mice. In this model, we demonstrated that colorectal PC preferred to colonize in visceral fats and $\mathrm{T}$ cells were altered dynamically with the development of colorectal PC.

\section{Abbreviations}

CRC: Colorectal cancer; PC: peritoneal carcinomatosis; HIPEC: hyperthermic intraperitoneal chemotherapy; DMEM: Dulbecco's modified Eagle's medium; TMMU: Third Military Medical University; RPMI: Roswell Park Memorial Institute; FBS: fetal bovine serum. 


\section{Acknowledgements}

This work was supported by the National Natural Science Foundation of China (81672693, to H.M.) and the Foundation and Frontier Research Project of Chongqing (cstc2017jcyjBX0071, to H.M.).

\section{Competing Interests}

The authors have declared that no competing interest exists.

\section{References}

1 Brenner H, Kloor M, Pox CP. Colorectal cancer. Lancet 2014; 383: 1490-1502.

2 Siegel R, Desantis C, Jemal A. Colorectal cancer statistics, 2014. CA: a cancer journal for clinicians 2014; 64: 104-117.

3 Baskaranathan S, Philips J, McCredden P, Solomon MJ. Free colorectal cancer cells on the peritoneal surface: correlation with pathologic variables and survival. Diseases of the colon and rectum 2004; 47: 2076-2079.

4 Koppe MJ, Boerman OC, Oyen WJ, Bleichrodt RP. Peritoneal carcinomatosis of colorectal origin: incidence and current treatment strategies. Annals of surgery 2006; 243: 212-222.

5 Solomon MJ, Egan M, Roberts RA, Philips J, Russell P. Incidence of free colorectal cancer cells on the peritoneal surface. Diseases of the colon and rectum 1997; 40: 1294-1298.

6 Sadeghi B, Arvieux C, Glehen O, Beaujard AC, Rivoire M, Baulieux J et al. Peritoneal carcinomatosis from non-gynecologic malignancies: results of the EVOCAPE 1 multicentric prospective study. Cancer 2000; 88: 358-363.

7 Elias D, Lefevre JH, Chevalier J, Brouquet A, Marchal F, Classe JM et al. Complete cytoreductive surgery plus intraperitoneal chemohyperthermia with oxaliplatin for peritoneal carcinomatosis of colorectal origin. Journal of clinical oncology : official journal of the American Society of Clinical Oncology 2009; 27: 681-685.

8 Sugarbaker PH. Peritonectomy procedures. Cancer treatment and research 2007; 134: 247-264.

9 Ceelen WP, Hesse U, de Hemptinne B, Pattyn P. Hyperthermic intraperitoneal chemoperfusion in the treatment of locally advanced intra-abdominal cancer. The British journal of surgery 2000; 87: 1006-1015.

10 Sugarbaker PH. Observations concerning cancer spread within the peritoneal cavity and concepts supporting an ordered pathophysiology. Cancer treatment and research 1996; 82: 79-100.

11 Arjona-Sanchez A, Medina-Fernandez FJ, Munoz-Casares FC, Casado-Adam A, Sanchez-Hidalgo JM, Rufian-Pena S. Peritoneal metastases of colorectal origin treated by cytoreduction and HIPEC: An overview. World journal of gastrointestinal oncology 2014; 6: 407-412.

12 Jayne DG, Fook S, Loi C, Seow-Choen F. Peritoneal carcinomatosis from colorectal cancer. The British journal of surgery 2002; 89: 1545-1550.

13 Nieman KM, Kenny HA, Penicka CV, Ladanyi A, Buell-Gutbrod R, Zillhardt MR et al. Adipocytes promote ovarian cancer metastasis and provide energy for rapid tumor growth. Nature medicine 2011; 17: 1498-1503.

14 Lengyel E. Ovarian cancer development and metastasis. The American journal of pathology 2010; 177: 1053-1064.

15 Ribas A, Wolchok JD. Cancer immunotherapy using checkpoint blockade. Science 2018; 359: 1350-1355.

16 Xiang W, Shi R, Kang X, Zhang X, Chen P, Zhang L et al. Monoacylglycerol lipase regulates cannabinoid receptor 2-dependent macrophage activation and cancer progression. Nat Commun 2018; 9: 2574.

17 June CH, O'Connor RS, Kawalekar OU, Ghassemi S, Milone MC. CAR T cell immunotherapy for human cancer. Science 2018; 359: 1361-1365.

18 Gajewski TF, Schreiber H, Fu YX. Innate and adaptive immune cells in the tumor microenvironment. Nature immunology 2013; 14: 1014-1022.

19 Jenkins MK, Khoruts A, Ingulli E, Mueller DL, McSorley SJ, Reinhardt RL et al. In vivo activation of antigen-specific CD4 $\mathrm{T}$ cells. Annual review of immunology 2001; 19: 23-45.

20 Hess Michelini R, Manzo T, Sturmheit T, Basso V, Rocchi M, Freschi M et al. Vaccine-instructed intratumoral IFN-gamma enables regression of autochthonous mouse prostate cancer in allogeneic T-cell transplantation. Cancer research 2013; 73: 4641-4652.

21 Gremonprez F, Descamps B, Izmer A, Vanhove C, Vanhaecke F, De Wever O et al. Pretreatment with VEGF(R)-inhibitors reduces interstitial fluid pressure, increases intraperitoneal chemotherapy drug penetration, and impedes tumor growth in a mouse colorectal carcinomatosis model. Oncotarget 2015; 6: 29889-29900.

22 Stoeltzing $\mathrm{O}$, Ahmad SA, Liu W, McCarty MF, Parikh AA, Fan F et al. Angiopoietin-1 inhibits tumour growth and ascites formation in a murine model of peritoneal carcinomatosis. Br J Cancer 2002; 87: 1182-1187.

23 Zhang W, Cui T, Liu L, Wu Q, Sun L, Li L et al. Improving Anti-Tumor Activity of Curcumin by Polymeric Micelles in Thermosensitive Hydrogel System in Colorectal Peritoneal Carcinomatosis Model. J Biomed Nanotechnol 2015; 11: 1173-1182.
24 Wu OJ, Gong CY, Luo ST, Zhang DM, Zhang S, Shi HS et al. AAV-mediated human PEDF inhibits tumor growth and metastasis in murine colorectal peritoneal carcinomatosis model. BMC Cancer 2012; 12: 129

25 Lee IK, Vansaun MN, Shim JH, Matrisian LM, Gorden DL. Increased metastases are associated with inflammation and matrix metalloproteinase-9 activity at incision sites in a murine model of peritoneal dissemination of colorectal cancer. J Surg Res 2013; 180: 252-259.

26 Facy O, Radais F, Ladoire S, Delroeux D, Tixier H, Ghiringhelli F et al. Comparison of hyperthermia and adrenaline to enhance the intratumoral accumulation of cisplatin in a murine model of peritoneal carcinomatosis. J Exp Clin Cancer Res 2011; 30: 4.

27 Mei LJ, Yang XJ, Tang L, Hassan AH, Yonemura Y, Li Y. Establishment and identification of a rabbit model of peritoneal carcinomatosis from gastric cancer. BMC Cancer 2010; 10: 124

28 Tokuda Y, Satoh Y, Fujiyama C, Toda S, Sugihara H, Masaki Z. Prostate cancer cell growth is modulated by adipocyte-cancer cell interaction. BJU international 2003; 91: 716-720.

29 Dirat B, Bochet L, Dabek M, Daviaud D, Dauvillier S, Majed B et al. Cancer-associated adipocytes exhibit an activated phenotype and contribute to breast cancer invasion. Cancer research 2011; 71: 2455-2465.

30 Okada M, Chikuma S, Kondo T, Hibino S, Machiyama H, Yokosuka T et al. Blockage of Core Fucosylation Reduces Cell-Surface Expression of PD-1 and Promotes Anti-tumor Immune Responses of T Cells. Cell reports 2017; 20: 1017-1028.

31 Sharma $\mathrm{P}$, Allison JP. The future of immune checkpoint therapy. Science 2015; 348: 56-61 